\title{
OPEN Risk factors for hemorrhoidal disease among healthy young and middle-aged Korean adults
}

\author{
Yun Soo Hong ${ }^{1,10}$, Kyung Uk Jung 2,10, Sanjay Rampal ${ }^{3}$, Di Zhao ${ }^{1}$, Eliseo Guallar ${ }^{1,4}$, \\ Seungho Ryu ${ }^{4,5,6}$, Yoosoo Chang $4,5,6$, Hyung Ook Kim², Hungdai Kim ${ }^{2}$, Ho-Kyung Chun ${ }^{2 \bowtie}$, \\ Chong II Sohn ${ }^{5,7}$, Hocheol Shin ${ }^{5,8}$ \& Juhee Cho ${ }^{1,4,5,9 \varpi}$
}

Hemorrhoidal disease is a highly prevalent anorectal condition causing substantial discomfort, disability, and decreased quality of life. Evidence on preventable risk factors for hemorrhoidal disease is limited. We conducted a cross-sectional study of 194,620 healthy men and women who completed a health screening exam including colonoscopy in 2011-2017. We evaluated potential risk factors of hemorrhoidal disease, including lifestyle factors, medical history, birth history, gastrointestinal symptoms, and anthropometric measurements. The prevalence of hemorrhoidal disease was $16.6 \%$, and it was higher in females than in males (17.2 vs. $16.3 \% ; P<0.001)$. Compared to men, the prevalence of hemorrhoidal disease was higher in parous women (adjusted odds ratio [OR] 1.06; 95\% confidence interval $[\mathrm{Cl}] 1.02-1.10$ ), and lower in nulliparous women (adjusted OR 0.92; $95 \% \mathrm{Cl} 0.86-$ $0.98)$. In the adjusted analyses, older age, female sex, smoking, overweight, and being hypertensive were independently associated with the presence of hemorrhoidal disease. The prevalence of hemorrhoidal disease was positively associated with body mass index and waist circumference in parous women. The prevalence of hemorrhoidal disease was higher in older age, females, eversmokers, and hypertensive participants. The association of excess adiposity with the prevalence of hemorrhoidal disease differed by sex and parity.

\author{
Abbreviations \\ BMI Body mass index \\ IPAQ-SF International physical activity questionnaire short form \\ MET Metabolic equivalent
}

Hemorrhoids are normal vascular cushions surrounding the distal rectum and anal canal that help maintain anal continence $^{1,2}$. The term hemorrhoids, however, commonly refers to pathological changes and distal displacement of hemorrhoidal tissue (hemorrhoidal disease), affecting nearly $40 \%$ of adults ${ }^{3,4}$. Hemorrhoidal disease can cause substantial discomfort, disability, and a decrease in quality of life ${ }^{5}$. It is also a major medical and socioeconomic problem, and represents a major burden to the health care system ${ }^{6,7}$.

Management of hemorrhoidal disease ranges from noninvasive medical approaches to invasive surgical procedures based on its disease grade ${ }^{8-14}$. Noninvasive methods do not reverse the underlying structural changes

\footnotetext{
${ }^{1}$ Departments of Epidemiology and Medicine, and Welch Center for Prevention, Epidemiology, and Clinical Research, Johns Hopkins University Bloomberg School of Public Health, Baltimore, MD, USA. ${ }^{2}$ Department of Surgery, Kangbuk Samsung Hospital, Sungkyunkwan University School of Medicine, 29 Saemunan-ro, Jongno-gu, Seoul 03181, South Korea. ${ }^{3}$ Department of Social and Preventive Medicine, Julius Centre University of Malaya, Faculty of Medicine, University of Malaya, Kuala, Lumpur, Malaysia. ${ }^{4}$ Department of Clinical Research Design \& Evaluation, SAIHST, Sungkyunkwan University, 81 Irwon-ro, Gangnam-gu, Seoul 06351, Republic of Korea. ${ }^{5}$ Center for Cohort Studies, Total Healthcare Center, Kangbuk Samsung Hospital, Sungkyunkwan University School of Medicine, Seoul, Republic of Korea. ${ }^{6}$ Department of Occupational and Environmental Medicine, Kangbuk Samsung Hospital, Sungkyunkwan University School of Medicine, Seoul, Republic of Korea. ${ }^{7}$ Division of Gastroenterology, Department of Internal Medicine and Gastrointestinal Cancer Center, Kangbuk Samsung Hospital, Sungkyunkwan University School of Medicine, Seoul, Korea. ${ }^{8}$ Department of Family Medicine, Kangbuk Samsung Hospital, Sungkyunkwan University School of Medicine, Seoul, South Korea. ${ }^{9}$ Center for Clinical Epidemiology, Samsung Medical Center, Seoul, South Korea. ${ }^{10}$ These authors contributed equally: Yun Soo Hong and Kyung Uk Jung. ${ }^{\varpi}$ email: hokyung.chun@samsung.com; jcho@skku.edu
} 


\begin{tabular}{|c|c|c|c|c|}
\hline & \multirow{2}{*}{\begin{tabular}{|l|} 
Overall, $n(\%)$ \\
$(n=194,620)$ \\
\end{tabular}} & \multicolumn{2}{|l|}{ Sex, $n(\%)$} & \multirow[b]{2}{*}{$P$} \\
\hline & & Women $(n=69,185)$ & Men $(n=125,435)$ & \\
\hline Age & & & & $<0.001$ \\
\hline 20 to 29 & $11,474(5.9)$ & $3,679(5.3)$ & $7,795(6.2)$ & \\
\hline 30 to 39 & $80,366(41.3)$ & $26,840(38.8)$ & $53,526(42.7)$ & \\
\hline 40 to 49 & $63,853(32.8)$ & $22,305(32.2)$ & $41,548(33.1)$ & \\
\hline 50 to 59 & $27,776(14.3)$ & $11,187(16.2)$ & $16,589(13.2)$ & \\
\hline 60 to 70 & $11,151(5.7)$ & $5,174(7.5)$ & $5,977(4.8)$ & \\
\hline Education $>12$ years & $145,517(74.8)$ & $44,850(64.8)$ & $100,667(80.3)$ & $<0.001$ \\
\hline Physical activity & & & & $<0.001$ \\
\hline Low & $92,330(47.4)$ & $37,063(53.6)$ & $55,267(44.1)$ & \\
\hline Moderate & $67,163(34.5)$ & $20,445(29.6)$ & $46,718(37.2)$ & \\
\hline High & $32,736(16.8)$ & $10,503(15.2)$ & $22,233(17.7)$ & \\
\hline Smoking & & & & $<0.001$ \\
\hline Never & $87,113(44.8)$ & $55,494(80.2)$ & $31,619(25.2)$ & \\
\hline Former & $53,466(27.5)$ & $4,642(6.7)$ & $48,824(38.9)$ & \\
\hline Current & $42,379(21.8)$ & $1,583(2.3)$ & $40,796(32.5)$ & \\
\hline Daily alcohol intake & & & & $<0.001$ \\
\hline None & $20,447(10.5)$ & $13,953(20.2)$ & $6,494(5.2)$ & \\
\hline$<5$ g/day & $56,053(28.8)$ & $29,793(43.1)$ & $26,260(20.9)$ & \\
\hline 5 to $<20 \mathrm{~g} /$ day & $60,666(31.2)$ & $13,538(19.6)$ & $47,128(37.6)$ & \\
\hline$\geq 20$ g/day & $45,816(23.5)$ & $3,680(5.3)$ & $42,136(33.6)$ & \\
\hline Body mass index $\left(\mathrm{kg} / \mathrm{m}^{2}\right)$ & $23.8(3.3)$ & $22.2(3.2)$ & $24.7(3.0)$ & $<0.001$ \\
\hline$\%$ Fat mass (\%) & $26.0(6.6)$ & $30.6(6.1)$ & $23.4(5.4)$ & $<0.001$ \\
\hline Waist circumference $(\mathrm{cm})$ & $83.1(9.4)$ & $76.9(8.5)$ & $86.6(8.0)$ & $<0.001$ \\
\hline Central obesity (\%) & $51,425(26.4)$ & $11,444(16.5)$ & $39,981(31.9)$ & $<0.001$ \\
\hline Diabetes & $10,254(5.3)$ & $2,429(3.5)$ & $7,825(6.2)$ & $<0.001$ \\
\hline Hypertension & $27,826(14.3)$ & $6,099(8.8)$ & $21,727(17.3)$ & $<0.001$ \\
\hline Dyslipidemia & $8,604(4.4)$ & $2,943(4.3)$ & $5,661(4.5)$ & 0.001 \\
\hline
\end{tabular}

Table 1. Characteristics of study participants. Values in the Table are number (\%) or mean (SD).

in the hemorrhoidal tissue, while invasive procedures have a relatively high frequency of complications and recurrence. Moreover, the severity and symptoms of hemorrhoidal disease tend to aggravate over time if it is not managed properly. Thus, the alternative approach to hemorrhoidal disease should be based on active prevention and early intervention, including dietary changes, medical treatment, and modification of potential risk factors ${ }^{15}$.

The evidence base on preventable risk factors for hemorrhoidal disease, however, is limited. Older age, female gender, lower socioeconomic status, lack of physical activity, pregnancy, alcohol consumption, constipation, diarrhea, prolonged abdominal straining, sedentary lifestyle, and obesity have been proposed as risk factors, but the findings are inconsistent across studies ${ }^{3,4,6,16-18}$. In addition, although previous studies have evaluated the associaiton of body mass index (BMI) with hemorrhoidal disease, the association of different measures of adiposity, including body fat distribution (central obesity) and body fat mass, and hemorrhoidal disease have not been explored. The objective of our study was thus to evaluate the risk factors associated with prevalent hemorrhoidal disease in a large study of healthy adults who underwent a health screening examination.

\section{Results}

The mean (SD) age of study participants was 42.2 (9.4) years. Men were younger, more physically active, more likely to be smokers, and more likely to have higher education levels, alcohol intake, BMI, and waist circumference, lower \% fat mass, and higher prevalence of diabetes, hypertension, and dyslipidemia compared to women (Table 1).

The prevalence of hemorrhoidal disease was $16.6 \%(n=32,347$; Table 2$)$, and it was higher in females than in males $(17.2$ vs. $16.3 \% ; P<0.001)$. Compared to men, parous women had a higher prevalence of hemorrhoidal disease (adjusted OR 1.06; 95\% CI 1.02-1.10), but nulliparous women had a lower prevalence than men (adjusted OR 0.92; 95\% CI 0.86-0.98). Among parous women, the prevalence of hemorrhoidal disease did not differ by type of birth (adjusted OR for ever given natural birth vs. cesarean section only $0.97 ; 95 \%$ CI $0.91-1.04$ ) or by number of births (adjusted ORs for 2 and 3 or more births vs. 1 birth 0.98 ; 95\% CI 0.93-1.04 and 0.97; 95\% CI $0.90-1.05$, respectively).

The prevalence of hemorrhoidal disease was higher in older participants, in those with lower education level, in those who were overweight or obese, or former smokers, and in those with diabetes, hypertension, or dyslipidemia. In adjusted analyses, increasing age, female sex, former or current smoking, being overweight, and being hypertensive were independently associated with the prevalence of hemorrhoidal disease (Table 2, Appendix Table 1). In analyses performed separately by sex and parity (Appendix Table 2), age and smoking were stronger 


\begin{tabular}{|c|c|c|c|c|c|}
\hline & $\begin{array}{l}\text { Hemorrhoidal disease, } \\
n(\%)\end{array}$ & $\begin{array}{l}\text { Crude odds ratio, OR } \\
\text { (95\% CI) }\end{array}$ & $P$ for trend & $\begin{array}{l}\text { Adjusted odds ratio*, OR } \\
(95 \% \text { CI })\end{array}$ & $P$ for trend \\
\hline Overall & $32,347(16.6)$ & & & & \\
\hline Age & & & $<0.001$ & & $<0.001$ \\
\hline 20 to 29 & $1,386(12.1)$ & 1.00 (ref) & & 1.00 (ref) & \\
\hline 30 to 39 & $11,152(13.9)$ & $1.17(1.10,1.24)$ & & $1.23(1.16,1.31)$ & \\
\hline 40 to 49 & $11,444(17.9)$ & $1.59(1.50,1.69)$ & & $1.62(1.53,1.72)$ & \\
\hline 50 to 59 & $5,908(21.3)$ & $1.97(1.85,2.09)$ & & $1.99(1.87,2.13)$ & \\
\hline 60 to 70 & $2,457(22.0)$ & $2.06(1.91,2.21)$ & & $2.06(1.91,2.22)$ & \\
\hline Sex & & & $<0.001$ & & 0.002 \\
\hline Men & $20,458(16.3)$ & 1.00 (ref) & & 1.00 (ref) & \\
\hline Women & $11,889(17.2)$ & $1.06(1.04,1.09)$ & & $1.06(1.02,1.09)$ & \\
\hline Sex / parity & & & $<0.001$ & & 0.002 \\
\hline Men & $20,458(16.3)$ & 1.00 (ref) & & 1.00 (ref) & \\
\hline Women, nulliparous & $1,432(13.5)$ & $0.80(0.76,0.85)$ & & $0.92(0.86,0.98)$ & \\
\hline Women, parous & $9,484(17.7)$ & $1.10(1.07,1.13)$ & & $1.06(1.02,1.10)$ & \\
\hline Natural birth, ever ${ }^{\dagger}$ & $7,001(17.7)$ & $1.10(1.07,1.14)$ & & $1.04(1.00,1.08)$ & \\
\hline C-section only ${ }^{\dagger}$ & $1,297(18.3)$ & $1.15(1.08,1.23)$ & & $1.08(1.01,1.16)$ & \\
\hline Education & & & $<0.001$ & & 0.75 \\
\hline$>12$ years & $23,598(16.2)$ & 1.00 (ref) & & 1.00 (ref) & \\
\hline$\leq 12$ years & $6,752(18.4)$ & $1.16(1.13,1.20)$ & & $1.01(0.97,1.04)$ & \\
\hline Physical activity & & & 0.30 & & 0.78 \\
\hline Low & $15,393(16.7)$ & 1.00 (ref) & & 1.00 (ref) & \\
\hline Moderate & $10,904(16.2)$ & $0.97(0.94,1.00)$ & & $0.97(0.94,0.99)$ & \\
\hline High & $5,624(17.2)$ & $1.04(1.00,1.07)$ & & $0.99(0.96,1.03)$ & \\
\hline Smoking status & & & 0.60 & & 0.28 \\
\hline Never & $14,309(16.4)$ & 1.00 (ref) & & 1.00 (ref) & \\
\hline Former & $9,083(17.0)$ & $1.04(1.01,1.07)$ & & $1.05(1.01,1.09)$ & \\
\hline Current & $6,956(16.4)$ & $1.00(0.97,1.03)$ & & $1.06(1.02,1.10)$ & \\
\hline Daily alcohol intake & & & 0.13 & & 0.34 \\
\hline None & $3,530(17.3)$ & 1.00 (ref) & & 1.00 (ref) & \\
\hline$<5$ g/day & $9,267(16.5)$ & $0.95(0.91,0.99)$ & & $1.01(0.97,1.06)$ & \\
\hline 5 to $<20 \mathrm{~g} /$ day & $9,854(16.2)$ & $0.93(0.89,0.97)$ & & $1.02(0.97,1.06)$ & \\
\hline$\geq 20 \mathrm{~g} /$ day & $7,632(16.7)$ & $0.96(0.92,1.00)$ & & $1.01(0.97,1.07)$ & \\
\hline Body mass index & & & 0.001 & & 0.75 \\
\hline Underweight & $1,047(15.2)$ & $0.92(0.86,0.99)$ & & $0.98(0.92,1.05)$ & \\
\hline Normal & $12,303(16.3)$ & 1.00 (ref) & & 1.00 (ref) & \\
\hline Overweight & $8,318(17.3)$ & $1.07(1.04,1.11)$ & & $1.04(1.01,1.08)$ & \\
\hline Obese & $10,679(16.7)$ & $1.03(1.00,1.06)$ & & $1.00(0.97,1.03)$ & \\
\hline Diabetes & & & $<0.001$ & & 0.07 \\
\hline No & $30,411(16.5)$ & 1.00 (ref) & & 1.00 (ref) & \\
\hline Yes & $1,918(18.7)$ & $1.16(1.11,1.23)$ & & $0.96(0.92,1.00)$ & \\
\hline Hypertension & & & $<0.001$ & & 0.001 \\
\hline No & $27,041(16.2)$ & 1.00 (ref) & & 1.00 (ref) & \\
\hline Yes & 5,244 (18.9) & $1.20(1.16,1.24)$ & & $1.04(1.02,1.07)$ & \\
\hline Dyslipidemia & & & $<0.001$ & & 0.93 \\
\hline No & $30,608(16.5)$ & 1.00 (ref) & & 1.00 (ref) & \\
\hline Yes & $1,724(20.0)$ & $1.27(1.20,1.34)$ & & $1.00(0.95,1.05)$ & \\
\hline
\end{tabular}

Table 2. Association between participant characteristics and the prevalence of hemorrhoidal disease. * Adjusted for age (20-29, 30-39, 40-49, 50-59, and 60-70 years), sex, year of visit, center, education ( $\leq 12$ and $>12$ years), physical activity (low, moderate, and high), smoking (never, former, and current), daily alcohol intake (none, $<5 \mathrm{~g} /$ day, 5 to $<20 \mathrm{~g} /$ day, and $\geq 20 \mathrm{~g} /$ day), BMI category (underweight, normal, overweight, and obese), and presence of diabetes, hypertension, and dyslipidemia. The ORs for sex / parity categories were obtained from a model with the same covariates but replacing sex with sex / parity categories. ${ }^{\dagger}$ Excluding participants without information on the mode of delivery. 


\begin{tabular}{|c|c|c|c|c|c|}
\hline & Hemorrhoids, $n$ (\%) & $\begin{array}{l}\text { Crude Odds Ratio, OR } \\
(95 \% \text { CI })\end{array}$ & $P$ for trend & $\begin{array}{l}\text { Adjusted Odds Ratio*, OR } \\
(95 \% \text { CI })\end{array}$ & $P$ for trend \\
\hline \% Fat mass & & & $<0.001$ & & $<0.001$ \\
\hline Quintile 1 & $6,184(15.9)$ & 1.00 (ref) & & 1.00 (ref) & \\
\hline Quintile 2 & $6,419(16.5)$ & $1.04(1.01,1.09)$ & & $0.99(0.95,1.02)$ & \\
\hline Quintile 3 & $6,432(16.5)$ & $1.05(1.01,1.09)$ & & $0.96(0.93,1.00)$ & \\
\hline Quintile 4 & $6,556(16.8)$ & $1.07(1.03,1.11)$ & & $0.96(0.92,1.00)$ & \\
\hline Quintile 5 & $6,756(17.4)$ & $1.11(1.07,1.15)$ & & $0.92(0.88,0.96)$ & \\
\hline Waist circumference & & & 0.26 & & 0.84 \\
\hline Quintile 1 & $6,098(15.9)$ & 1.00 (ref) & & 1.00 (ref) & \\
\hline \begin{tabular}{|l|} 
Quintile 2 \\
\end{tabular} & $6,606(16.9)$ & $1.07(1.03,1.11)$ & & $1.06(1.02,1.10)$ & \\
\hline Quintile 3 & $6,681(17.2)$ & $1.09(1.05,1.13)$ & & $1.07(1.02,1.11)$ & \\
\hline Quintile 4 & $6,607(16.8)$ & $1.07(1.03,1.11)$ & & $1.05(1.00,1.09)$ & \\
\hline Quintile 5 & 6,355 (16.3) & $1.03(0.99,1.07)$ & & $1.02(0.97,1.07)$ & \\
\hline Central obesity & & & 0.40 & & 0.49 \\
\hline No & $23,739(16.6)$ & 1.00 (ref) & & 1.00 (ref) & \\
\hline Yes & $8,608(16.7)$ & $1.01(0.98,1.04)$ & & $0.99(0.96,1.02)$ & \\
\hline
\end{tabular}

Table 3. Association between alternative markers of adiposity and the prevalence of hemorrhoidal disease. * Adjusted models included age (20-29, 30-39, 40-49, 50-59, and 60-70 years), sex, year of visit, center, education ( $\leq 12$ and $>12$ years), physical activity (low, moderate, and high), smoking (never, former, and current), daily alcohol intake (none, $<5 \mathrm{~g} /$ day, 5 to $<20 \mathrm{~g} /$ day, and $\geq 20 \mathrm{~g} /$ day), and presence of diabetes, hypertension, and dyslipidemia, plus one of the following markers of excess adiposity: $\%$ fat mass $\%$ categorized in quintiles $(<20.34,20.35-23.75,23.75-27.08,27.08-31.58, \geq 31.58 \%)$, waist circumference categorized in quintiles $(<74.7,74.7-80.5,80.5-85.1,85.2-90.5$, and $\geq 90.5 \mathrm{~cm})$, and central obesity (no vs. yes).

risk factors in men than in nulliparous women and in parous women ( $P$ interaction $<0.001$ and 0.30 , respectively). In addition, being overweight or obese was a particularly strong risk factor in parous women ( $P$ interaction 0.07 ), and having dyslipidemia was the strongest risk factor in nulliparous women ( $P$ interaction 0.007$)$.

When we studied measures of adiposity other than BMI (Table 3 ), \% fat mass showed an inverse association ( $P$ for trend $<0.001$ ) with the prevalence of hemorrhoidal disease in fully adjusted models, while there was no clear trend with waist circumference and central obesity. In sex and parity specific models (Appendix Table 3), there was an inverse association of $\%$ fat mass with the prevalence of hemorrhoidal disease was inverse in men and nulliparous women ( $P$ interaction 0.46$)$, and a positive association of waist circumference in parous women $(P$ interaction $<0.001)$. Central adiposity showed an inverse association with the prevalence of hemorrhoidal disease in men (adjusted OR 0.96; 95\% CI 0.93-1.00), and a positive association in parous women (adjusted OR 1.08 ; $95 \%$ CI 1.02-1.15).

Among self-reported digestive and lower gastrointestinal symptoms (Table 4), the presence of red blood in stools (OR 1.54; 95\% CI 1.46-1.63), constipation (OR 1.10; 95\% CI 1.06-1.14), narrow caliber stools (OR 1.05; 95\% CI 1.01-1.10), and tenesmus (OR 1.05, 95\% CI 1.02-1.09) were significantly associated with an increased prevalence of hemorrhoidal disease, while difficulty in digesting food (OR 0.96; 95\% CI 0.93-0.99) was associated with a lower prevalence of hemorrhoidal disease. The associations between symptoms and the prevalence of hemorrhoidal disease were similar across sex and history of parity groups (Appendix Table 4).

\section{Discussion}

In this study of healthy Korean adults participating in a health screening program with a complete colonoscopic examination, increasing age, female sex, smoking, presence of hypertension, history of childbirth, and presence of fresh blood in stools, constipation, narrow caliber stool, or tenesmus were associated with hemorrhoidal disease. The association between adiposity and the prevalence of hemorrhoidal disease was complex and differed by sex and parity.

The overall prevalence of hemorrhoidal disease in our study was $16.6 \%$. The prevalence of hemorrhoidal disease ranged from 4.4 to $88 \%^{19-21}$, depending on the study population and the methods of ascertainment (selfreport of symptoms vs. physician diagnosis), but even recent studies using screening colonoscopy reported a wide range of prevalences $(20.8 \text { to } 38.2 \%)^{3,6,22}$.

In our study, the prevalence of hemorrhoidal disease increased progressively with age, with the highest prevalence observed in the oldest age group. Some studies reported the highest prevalence of hemorrhoid among middle aged men and women, but these estimates were based on a smaller sample size and self-reports or physician diagnosis of hemorrhoidal disease ${ }^{3}$, which may be subject to misclassification ${ }^{16}$.

Parous women had a significantly higher prevalence of hemorrhoidal disease compared to men and to nulliparous women, likely due to mechanical changes of the pelvic floor because of increased intra-abdominal pressure, pelvic venous congestion, and damage during labor, and to hormonal factors affecting gastrointestinal motility during pregnancy ${ }^{23}$. In previous studies, the prevalence of hemorrhoidal disease was highest in the 2 nd and 3rd trimester of pregnancy and the incidence was $\sim 24 \%$ during the first 6 months of delivery ${ }^{23-26}$. Consistent 


\begin{tabular}{|c|c|c|c|c|c|}
\hline & Hemorrhoids, $n$ (\%) & $\begin{array}{l}\text { Crude Odds Ratio, OR } \\
\text { (95\% CI) }\end{array}$ & $P$ & $\begin{array}{l}\text { Adjusted Odds Ratio*, OR } \\
\text { (95\% CI) }\end{array}$ & $P$ \\
\hline Difficulty swallowing & $184(16.2)$ & $0.97(0.83,1.14)$ & 0.73 & $0.92(0.78,1.07)$ & 0.28 \\
\hline Acid regurgitation & $3,110(17.1)$ & $1.04(1.00,1.08)$ & 0.05 & $1.01(0.97,1.05)$ & 0.69 \\
\hline Nausea and/or vomiting & $1,055(15.5)$ & $0.92(0.86,0.98)$ & 0.01 & $0.96(0.90,1.03)$ & 0.29 \\
\hline Difficulty in digestion & $6,150(15.8)$ & $0.93(0.90,0.95)$ & $<0.001$ & $0.96(0.93,0.99)$ & 0.01 \\
\hline Heartburn & $4,270(16.6)$ & $1.00(0.96,1.04)$ & 0.97 & $0.98(0.95,1.02)$ & 0.40 \\
\hline Tarry stools & $690(16.2)$ & $0.97(0.89,1.05)$ & 0.47 & $0.97(0.90,1.06)$ & 0.53 \\
\hline Fresh blood in stools & $1,820(21.8)$ & $1.42(1.35,1.50)$ & $<0.001$ & $1.54(1.46,1.63)$ & $<0.001$ \\
\hline Frequent diarrhea & $4,324(15.6)$ & $0.92(0.88,0.95)$ & $<0.001$ & $0.97(0.93,1.00)$ & 0.06 \\
\hline Constipation & $3,866(17.8)$ & $1.09(1.05,1.14)$ & $<0.001$ & $1.10(1.06,1.14)$ & $<0.001$ \\
\hline Narrow caliber stools & $2,554(17.6)$ & $1.08(1.03,1.13)$ & 0.001 & $1.05(1.01,1.10)$ & 0.02 \\
\hline Lump or mass in the abdomen & $498(16.5)$ & $0.99(0.90,1.10)$ & 0.92 & $0.99(0.90,1.09)$ & 0.87 \\
\hline Frequent abdominal pain & $2,093(15.9)$ & $0.94(0.90,0.99)$ & 0.02 & $1.01(0.96,1.06)$ & 0.72 \\
\hline Tenesmus & $5,269(17.1)$ & $1.04(1.01,1.07)$ & 0.02 & $1.05(1.02,1.09)$ & 0.004 \\
\hline
\end{tabular}

Table 4. Association between self-reported gastrointestinal symptoms and the prevalence of hemorrhoidal disease. ${ }^{*}$ Adjusted for age (20-29, 30-39, 40-49, 50-59, and 60-70 years), sex, year of visit, center, education ( $\leq 12$ and $>12$ years), physical activity (low, moderate, and high), smoking (never, former, and current), daily alcohol intake (none, $<5 \mathrm{~g} /$ day, 5 to $<20 \mathrm{~g} /$ day, and $\geq 20 \mathrm{~g}$ /day), BMI category (underweight, normal, overweight, and obese), and presence of diabetes, hypertension, and dyslipidemia.

with previous studies ${ }^{3,6,23,24}$, the number of childbirths was not associated with increased risk of hemorrhoidal disease in our study.

Smoking was also associated with an increased prevalence of hemorrhoidal disease, possibly by promoting systemic inflammation and by its effects on collagen metabolism ${ }^{16,27-31}$. Although, the association between hypertension and hemorrhoidal disease has not been described in other studies, hypertension facilitates vascular injury through oxidative stress and inflammation, and may, thereby, further damage adjacent connective tissue ${ }^{32}$.

The association of excess adiposity with hemorrhoidal disease is controversial ${ }^{6,16}$. In our study, the risk of hemorrhoidal disease did not increase linearly with increasing BMI, but overweight women had an increased prevalence of hemorrhoidal disease. In addition, \% fat mass was inversely associated with prevalence of hemorrhoidal disease in men and nulliparous women, while waist circumference was associated with a higher prevalence of hemorrhoidal disease only in parous women. The association of excess adiposity and its distribution with the risk of hemorrhoidal disease is thus complex, and may depend on sex, parity, and other characteristics. Additional research is needed to confirm our findings in other populations.

Among various self-reported gastrointestinal symptoms, fresh blood in stools, constipation, narrow caliber stools, and tenesmus were associated with the presence of hemorrhoidal disease. These symptoms may be a consequence of hemorrhoidal disease and not an antecedent factor. Constipation can cause hemorrhoidal disease by increasing intra-abdominal pressure, resulting in hemorrhoidal plexus engorgement, and in excessive straining during defecation leading to shearing forces on the anal cushions ${ }^{3,6,16,33}$.

There are several limitations to our study. As a cross-sectional study, we cannot assume temporality or causality between the risk factors and hemorrhoidal disease. Although a detailed examination of the anal and rectal areas is the standard-of-care in colonoscopy, the severity of hemorrhoidal disease, such as the Goligher classification, was not collected. Moreover, as the study population was participants undergoing a routine screening exam and not an evaluation for hemorrhoidal disease, an anoscopy was not performed in addition to a routine colonoscopy. In addition, bowel preparation prior to colonoscopy may increase congestion of the internal hemorrhoidal plexus and result in over-diagnosis or over-staging. Furthermore, the definition of hemorrhoidal disease in our study included any internal and/or external hemorrhoidal disease assessed by colonoscopy and may have included enlargement/bleeding of external hemorrhoidal plexus, which is considered to have a different pathology. The heterogeneity in the definition of hemorrhoidal disease around internal and external hemorrhoidal disease in the literature and in clinical practice may also lead to inconsistent results across studies. Moreover, because the questionnaire in this study was not designed specifically to screen for hemorrhoidal disease or to assess it severity, we were also unable to evaluate the severity of hemorrhoid-related anorectal symptoms. However, some of the anorectal symptoms included in the questionnaire, such as fresh blood in stools, constipation, and narrow caliber stools, were highly associated with the presence of hemorrhoidal disease.

In addition, study participants were predominantly young and middle-aged apparently healthy Korean adults without any previous history of colorectal disease. Therefore, the associations observed may not be generalized to other populations and race / ethnicity groups who have different genetic or lifestyle factors that might influence the presence of hemorrhoidal disease, or to populations with previous colorectal conditions.

Our study also has major strengths. We used a well-characterized cohort with high-quality information on multiple potential risk factors. In addition, this is the largest study to date evaluating risk factors for hemorrhoidal disease using a complete colonoscopic examination to ascertain the presence of disease. This approach is less likely to miss participants with asymptomatic hemorrhoidal disease and participants who might hide the disease 


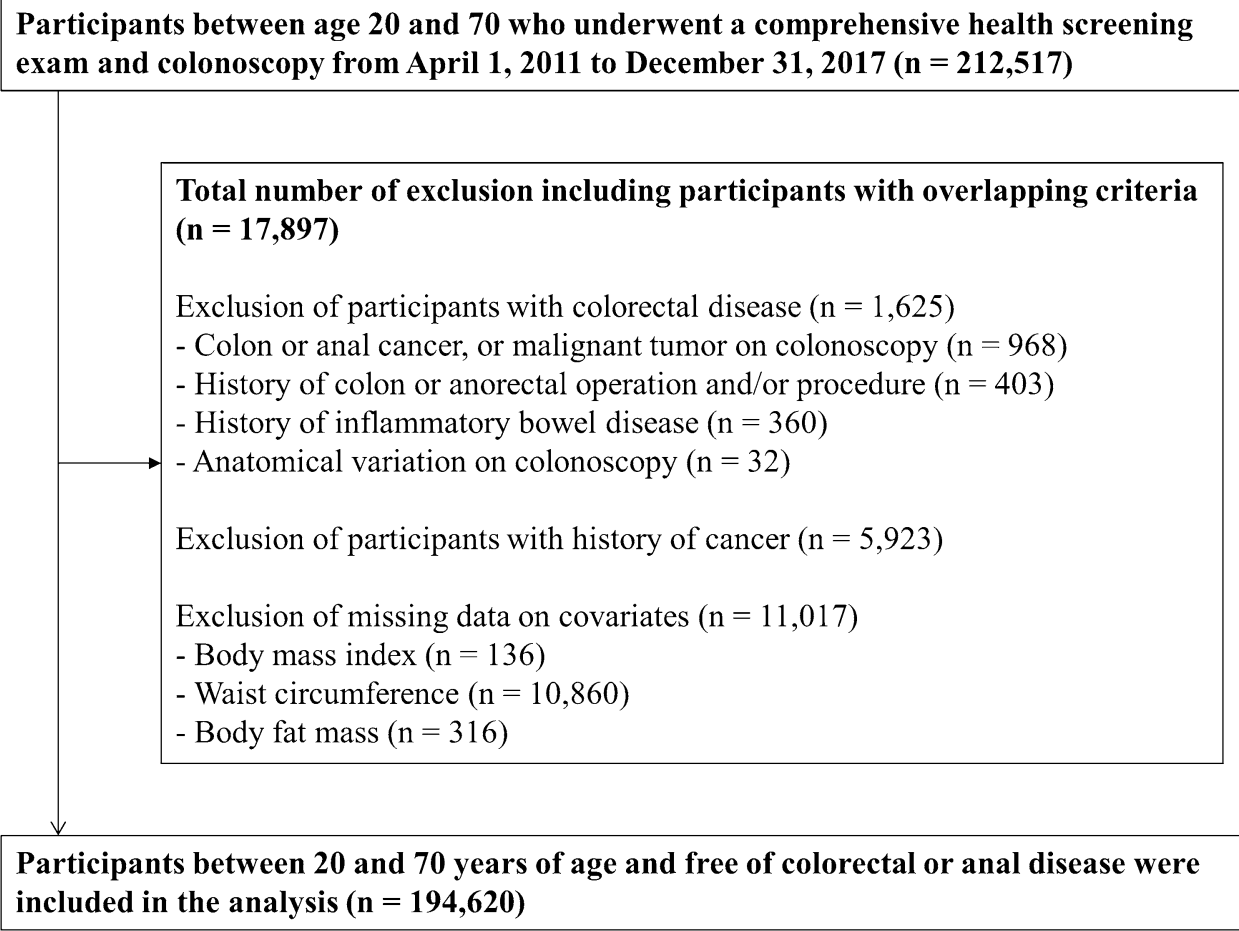

Figure 1. Flowchart of study participants.

due to embarrassment, which are major limitations of using claims-based data and self-reported questionnaire to define the presence of hemorrhoidal disease.

In this study, we found that the prevalence of hemorrhoidal disease increased with age and it was higher in parous women compared to men and to nulliparous women. Participants who ever smoked, who were hypertensive, and who reported fresh blood in stools or constipation also had an increased prevalence of hemorrhoidal disease. The association of excess adiposity with the prevalence of hemorrhoidal disease was complex and differed by sex and parity. Further epidemiological studies with longitudinal design are needed to better understand how these factors interact in the development of hemorrhoidal disease.

\section{Methods}

Study population. The Kangbuk Samsung Health Study is a cohort of adult men and women who underwent a comprehensive annual or biennial health screening exam at the Kangbuk Samsung Hospital Total Healthcare Screening Centers in Seoul and Suwon, South Korea ${ }^{34,35}$. Korean government mandates that employers provide regular health screening exams to their employees free of charge by the Industrial Safety and Health Law. Over $80 \%$ of the study participants in the Kangbuk Samsung Health Study are employees (or their relatives) of companies that contracted the routine health screening exams with the Kangbuk Samsung Hospital. The rest of the participants voluntarily purchased the health screening exams. For this study, we included participants 20 to 70 years of age who completed a health exam and had undergone at least one colonoscopy as part of routine health screening between April 2011 and December 2017, when the colonoscopy data in the Kangbuk Samsung Health Study was available $(n=212,517)$.

We excluded participants with colorectal disease $(n=1,625)$, including colorectal or anal cancer findings on colonoscopy $(n=968)$, a history of colorectal or anorectal cancer operation and/or procedure $(n=403)$, a history of inflammatory bowel disease $(n=360)$, and anatomical variation on colonoscopy $(n=32)$; participants with a history of cancer $(n=5,923)$; and participants with missing data on BMI, waist circumference, or body fat mass $(n=11,017)$. The final sample size was 194,620 participants $(125,4365$ men and 69,185 women; Fig. 1$)$. For participants who attended more than one screening exam during the study period, we included only data from the initial exam in the analysis.

The study was approved by the Institutional Review Board of the Kangbuk Samsung Hospital, which waived the requirement for informed consent because we used only de-identified information obtained as part of routine health screening exams. All research methods were performed in accordance with the relevant guidelines and regulations.

Measurements. In each screening exam, study participants provided information on their medical history, smoking habits, alcohol consumption, physical activity, and education level through a standardized selfadministered questionnaire. Smoking habits were categorized as never, former, or current. Alcohol consumption was calculated in $\mathrm{g} /$ day and further categorized into none, $<5 \mathrm{~g} /$ day, 5-20 g/day, and $\geq 20 \mathrm{~g} /$ day. We used the 
validated Korean version of the International Physical Activity Questionnaire Short Form (IPAQ-SF) to assess the frequency and duration of walking, moderate-intensity, and vigorous-intensity physical activity for more than 10 consecutive minutes across all daily activities over a 7-day span ${ }^{36}$. We then calculated an activity score as the number of minutes spent on each type of physical activity per week multiplied by the metabolic equivalent $(\mathrm{MET})$ of each activity and categorized the level of physical activity into low, moderate, and high ${ }^{37}$. Education level was defined as $\leq 12$ or $>12$ years of education.

Height, weight, waist circumference, and body composition were measured by trained staff members. BMI was calculated as weight in kilograms divided by height in meters squared $\left(\mathrm{kg} / \mathrm{m}^{2}\right)$. BMI was classified according to the criteria proposed for Asian populations by the World Health Organization: ${ }^{38}$ underweight $15.0-18.4 \mathrm{~kg} /$ $\mathrm{m}^{2}$; normal 18.5-22.9 kg/m²; overweight $23.0-24.9 \mathrm{~kg} / \mathrm{m}^{2}$; obese $\geq 25.0 \mathrm{~kg} / \mathrm{m}^{2}$. Waist circumference was measured at the midpoint between the bottom of the rib cage and the top of the iliac crest to the nearest $0.1 \mathrm{~cm}$ in a standing position with weight equally distributed on both feet, arms at their sides, and head facing forward. Central obesity was defined by the guidelines suggested for Koreans $(\geq 90 \mathrm{~cm} \text { in men and } \geq 85 \mathrm{~cm} \text { in women })^{39}$. $\%$ fat mass was measured using a multi-frequency bioimpedance analyzer (Inbody 3.0 and Inbody 720, Biospace Co., Seoul, Republic of Korea). Hypertension was defined as a systolic blood pressure $\geq 140 \mathrm{mmHg}$, a diastolic blood pressure $\geq 90 \mathrm{mmHg}$, a self-reported physician diagnosis, or current use of antihypertensive medication.

Clinical chemistry measurements were performed in venous blood samples obtained after more than $10 \mathrm{~h}$ of fasting. Diabetes was defined as a fasting serum glucose $\geq 126 \mathrm{mg} / \mathrm{dl}$, a self-reported physician diagnosis, or current use of insulin or other hypoglycemic agents. Dyslipidemia was defined as a self-reported physician diagnosis, or current use of lipid lowering medication.

Prior to colonoscopy, participants were asked for the presence of any digestive or lower gastrointestinal symptoms from a list of 13 symptoms which were created based on clinical significance and frequency of report in previous health surveys at the center. The list included nausea and/or vomiting, acid regurgitation, heartburn, difficulty in swallowing, difficulty in digesting, frequent abdominal pain, constipation, frequent diarrhea, narrowcaliber stools, fresh blood in stools, tarry stools, tenesmus, and lumps or masses in the abdomen. For female participants, we also assessed the history of ever being pregnant, number of childbirths (parity), and mode of delivery for each birth (natural birth or cesarean section).

The presence of hemorrhoidal disease was assessed during colonoscopy performed as part of routine screening exams. Although colonoscopy was used to diagnose the presence of hemorrhoidal disease, the purpose of the exam was to screen for colorectal neoplasms and/or other colorectal conditions. Rectal examination was performed prior to insertion of the colonoscope. Colonoscopies was performed by experienced gastroenterologists using EVIS LUCERA CV-260 colonoscopes (Olympus Co., Tokyo, Japan). We used a polyethylene glycol solution (Taejoon Pharm. Inc., Seoul, Republic of Korea) of $4 \mathrm{~L}$ for bowel cleansing. The presence of hemorrhoidal disease was defined as morphological presence of internal and/or external hemorrhoidal disease assessed by a colonoscopic exam. Internal hemorrhoidal disease was defined as the presence of an enlarged vein above the dentate line on retroflexion of the colonoscope. External hemorrhoidal disease was defined as the presence of an enlarged vein at or below the dentate line.

Statistical analysis. The association between potential risk factors and the prevalence of hemorrhoidal disease was evaluated using logistic regression. We estimated the adjusted odds ratios (OR) and their 95\% confidence intervals (CIs) for the prevalence of hemorrhoidal disease in models including age category (20-29, $30-39,40-49,50-59$, and 60-70 years), sex, education (>12 and $\leq 12$ years), smoking (never, former, and current), alcohol intake (none, $<5 \mathrm{~g} /$ day, 5-20 g/day, and $\geq 20 \mathrm{~g} /$ day), physical activity (low, moderate, and high), BMI category (underweight, normal, overweight, and obese), and a history of diabetes, hypertension, and dyslipidemia. We also evaluated the independent association between different markers of adiposity (\% fat mass, waist circumference, and central obesity), as well as the association between sex, parity, and mode of delivery and hemorrhoidal disease. In addition, we evaluated the association between each of the 13 gastrointestinal symptoms and hemorrhoidal disease in the fully adjusted model. Linear trends of the associations were also evaluated by using the median values for each category as a continuous variable in the regression models.

All statistical analyses were performed with Stata version 15.0 (StataCorp LP, College Station, TX, USA). All $P$ values reported in the study are two-sided and $P$ values $<0.05$ were considered statistically significant.

Ethics approval. The study was approved by the Institutional Review Board of the Kangbuk Samsung Hospital, which waived the requirement for informed consent because we used only de-identified information obtained as part of routine health screening exams.

\section{Code availability}

Upon request to the authors of the paper.

Received: 22 October 2020; Accepted: 6 December 2021

Published online: 07 January 2022

\section{References}

1. Gallo, G., Sacco, R. \& Sammarco, G. Epidemiology of hemorrhoidal disease. In Hemorrhoids (eds Ratto, C. et al.) 3-7 (Springer, 2018).

2. Lohsiriwat, V. Anatomy, physiology, and pathophysiology of hemorrhoids. In Hemorrhoids (eds Ratto, C. et al.) 9-17 (Springer, 2018).

3. Riss, S. et al. The prevalence of hemorrhoids in adults. Int. J. Colorect. Dis. 27(2), 215-220. https://doi.org/10.1007/s00384-0111316-3 (2012). 
4. Pata, F. et al. Anatomy, physiology and pathophysiology of haemorrhoids. Rev. Recent Clin. Trials 16(1), 75-80. https://doi.org/ $10.2174 / 1574887115666200406115150$ (2021).

5. Senagore AJ. Surgical management of hemorrhoids. J. Gastrointest. Surg. Off. J. Soc. Surg. Aliment. Tract 2002;6(3):295-298

6. Peery, A. F. et al. Risk factors for hemorrhoids on screening colonoscopy. PLoS ONE 10(9), e0139100. https://doi.org/10.1371/ journal.pone.0139100 (2015)

7. Yang JY, Peery AF, Lund JL, Pate V, Sandler RS. Burden and cost of outpatient hemorrhoids in the United States employer-insured population, 2014. Am. J. Gastroenterol. 2019. DOI: https://doi.org/10.14309/ajg.0000000000000143.

8. Altomare, D. F. \& Giannini, I. Pharmacological treatment of hemorrhoids: a narrative review. Exp. Opin. Pharmacoth. 14(17), 2343-2349. https://doi.org/10.1517/14656566.2013.836181 (2013).

9. Song, S. G. \& Kim, S. H. Optimal treatment of symptomatic hemorrhoids. J. Korean Soc. Coloproctol. 27(6), 277-281. https://doi. org $/ 10.3393 /$ jksc.2011.27.6.277 (2011).

10. Gallo, G. et al. Consensus statement of the Italian society of colorectal surgery (SICCR): management and treatment of hemorrhoidal disease. Tech. Coloproctol. 24(2), 145-164. https://doi.org/10.1007/s10151-020-02149-1 (2020).

11. van Tol, R. R. et al. European society of coloproctology: guideline for haemorrhoidal disease. Colorectal. Dis. 22(6), 650-662. https://doi.org/10.1111/codi.14975 (2020).

12. Picciariello, A. et al. Classifications and clinical assessment of haemorrhoids: the proctologist's corner. Rev. Recent Clin. Trials 16(1), 10-16. https://doi.org/10.2174/1574887115666200312163940 (2021).

13. Stratta, E., Gallo, G. \& Trompetto, M. Conservative treatment of hemorrhoidal disease. Rev. Recent Clin. Trials 16(1), 87-90. https:// doi.org/10.2174/1574887115666201021150144 (2021).

14. Sobrado Júnior CW, Obregon CA, AHDS ESJ, Sobrado LF, Nahas SC, Cecconello I. A new classification for hemorrhoidal disease: the creation of the "BPRST" staging and its application in clinical practice. Ann. Coloproctol. 2020;36(4):249-255. doi: https://doi. org/10.3393/ac.2020.02.06.

15. Brisinda G. How to treat haemorrhoids. Prevention is best; haemorrhoidectomy needs skilled operators. BMJ (Clinical research ed) 2000;321(7261):582-3. (http://www.ncbi.nlm.nih.gov/pmc/articles/PMC1118483/pdf/582.pdf).

16. Lee, J. H., Kim, H. E., Kang, J. H., Shin, J. Y. \& Song, Y. M. Factors associated with hemorrhoids in korean adults: korean national health and nutrition examination survey. Korean J. Family Med. 35(5), 227-236. https://doi.org/10.4082/kjfm.2014.35.5.227 (2014).

17. Riss S, Weiser FA, Schwameis K, Mittlbock M, Stift A. Haemorrhoids, constipation and faecal incontinence: is there any relationship? Colorectal Dis. Off. J. Assoc. Coloproctol. Great Britain Ireland 2011;13(8):e227-33. doi: https://doi.org/10.1111/j.1463-1318. 2011.02632.x.

18. Johanson JF. Association of hemorrhoidal disease with diarrheal disorders: potential pathogenic relationship? Diseases of the colon and rectum 1997;40(2):215-9; discussion 219-21. (In eng).

19. Lohsiriwat, V. Hemorrhoids: from basic pathophysiology to clinical management. World J. Gastroenterol. WJG 18(17), $2009-2017$. https://doi.org/10.3748/wjg.v18.i17.2009 (2012).

20. Kaidar-Person, O., Person, B. \& Wexner, S. D. Hemorrhoidal disease: a comprehensive review. J. Am. College Surg. 204(1), 102-117. https://doi.org/10.1016/j.jamcollsurg.2006.08.022 (2007).

21. Madoff, R. D. \& Fleshman, J. W. American Gastroenterological Association technical review on the diagnosis and treatment of hemorrhoids. Gastroenterology 126(5), 1463-1473 (2004).

22. Akere, A., Oke, T. O. \& Otegbayo, J. A. Colonoscopy at a tertiary healthcare facility in Southwest Nigeria: spectrum of indications and colonic abnormalities. Ann. Afr. Med. 15(3), 109-113. https://doi.org/10.4103/1596-3519.188889 (2016).

23. Avsar, A. F. \& Keskin, H. L. Haemorrhoids during pregnancy. J. Obstet. Gynaecol. 30(3), 231-237. https://doi.org/10.3109/01443 $610903439242(2010)$.

24. Poskus, T. et al. Haemorrhoids and anal fissures during pregnancy and after childbirth: a prospective cohort study. BJOG 121(13), 1666-1671. https://doi.org/10.1111/1471-0528.12838 (2014).

25. Wall, L. L. Pelvis, posture and protrusion: evolution and haemorrhoids. BJOG 121(13), 1672. https://doi.org/10.1111/1471-0528. 12840 (2014).

26. Borders, N. After the afterbirth: a critical review of postpartum health relative to method of delivery. J. Midwifery Women's Health 51(4), 242-248. https://doi.org/10.1016/j.jmwh.2005.10.014 (2006).

27. La Vecchia, C., Pagano, R., Negri, E. \& Decarli, A. Smoking and prevalence of disease in the 1983 Italian National Health Survey. Int. J. Epidemiol. 17(1), 50-55 (1988).

28. Kruger K, Dischereit G, Seimetz M, Wilhelm J, Weissmann N, Mooren FC. Time course of cigarette smoke-induced changes of systemic inflammation and muscle structure. Am. J. Physiol. Lung Cell Mol. Physiol. 2015;309(2):L119-28. doi: https://doi.org/ 10.1152/ajplung.00074.2015.

29. Overbeek, S. A. et al. Cigarette smoke-induced collagen destruction; key to chronic neutrophilic airway inflammation?. PLoS ONE 8(1), e55612. https://doi.org/10.1371/journal.pone.0055612 (2013).

30. Kisli, E., Kemik, A., Sumer, A. \& Kemik, O. Matrix metalloproteinases in pathogenesis of hemorrhoidal disease. Am. Surg. 79(11), 1181-1184 (2013).

31. Willis, S., Junge, K., Ebrahimi, R., Prescher, A. \& Schumpelick, V. Haemorrhoids - a collagen disease?. Colorectal Dis. 12(12), 1249-1253. https://doi.org/10.1111/j.1463-1318.2009.02010.x (2010).

32. Briones, A. M. \& Touyz, R. M. Oxidative stress and hypertension: current concepts. Curr. Hypertens. Rep. 12(2), 135-142. https:// doi.org/10.1007/s11906-010-0100-z (2010).

33. Johanson, J. F. \& Sonnenberg, A. Constipation is not a risk factor for hemorrhoids: a case-control study of potential etiological agents. Am. J. Gastroenterol. 89(11), 1981-1986 (1994).

34. Zhang, Y. et al. Thyroid hormone levels and incident chronic kidney disease in euthyroid individuals: the Kangbuk Samsung Health Study. Int. J. Epidemiol. 43(5), 1624-1632. https://doi.org/10.1093/ije/dyu126 (2014).

35. Chang, Y. et al. Metabolically-healthy obesity and coronary artery calcification. J. Am. College Cardiol. 63(24), 2679-2686. https:// doi.org/10.1016/j.jacc.2014.03.042 (2014).

36. Oh, J. Y., Yang, Y. J., Kim, B. S. \& Kang, J. H. Validity and reliability of Korean version of International Physical Activity Questionnaire (IPAQ) short form. J. Korean Acad. Family Med. 28(7), 532-541 (2007).

37. Committee IR. Guidelines for data processing and analysis of the International Physical Activity Questionnaire (IPAQ)-short and long forms. 2005.

38. Wen, C. P. et al. Are Asians at greater mortality risks for being overweight than Caucasians? Redefining obesity for Asians. Public Health Nutr. 12(4), 497-506. https://doi.org/10.1017/s1368980008002802 (2009).

39. Han, J. H. et al. Visceral adipose tissue as a predictor for metabolic risk factors in the Korean population. Diab. Med. J. Br. Diab. Assoc. 25(1), 106-110. https://doi.org/10.1111/j.1464-5491.2007.02317.x (2008).

\section{Author contributions}

Drs. K.U.J., H.O.K., H.K., H.-K.C., C.I.S. made contributions to the conception and design and interpretation of the data. Drs. Y.S.H., S.R., E.G., and J.C. contributed to the conception and design of the study, analysis and interpretation of data, and drafting the manuscript. Drs. S.R., Y.C., and H.S. had major contributions to the acquisition of data, interpretation of the data, and supervision of the research group. All authors contributed 
substantially to revising the manuscript and providing intellectual content and provided final approval of the version to be published.

\section{Competing interests}

The authors declare no competing interests.

\section{Additional information}

Supplementary Information The online version contains supplementary material available at https://doi.org/ 10.1038/s41598-021-03838-Z.

Correspondence and requests for materials should be addressed to H.-K.C. or J.C.

Reprints and permissions information is available at www.nature.com/reprints.

Publisher's note Springer Nature remains neutral with regard to jurisdictional claims in published maps and institutional affiliations.

(c) (i) Open Access This article is licensed under a Creative Commons Attribution 4.0 International License, which permits use, sharing, adaptation, distribution and reproduction in any medium or format, as long as you give appropriate credit to the original author(s) and the source, provide a link to the Creative Commons licence, and indicate if changes were made. The images or other third party material in this article are included in the article's Creative Commons licence, unless indicated otherwise in a credit line to the material. If material is not included in the article's Creative Commons licence and your intended use is not permitted by statutory regulation or exceeds the permitted use, you will need to obtain permission directly from the copyright holder. To view a copy of this licence, visit http://creativecommons.org/licenses/by/4.0/.

(C) The Author(s) 2022 